\title{
Intersection Theory on the Moduli Space of Curves and the Matrix Airy Function*
}

\author{
Maxim Kontsevich \\ Max-Planck Institut für Mathematik, Gottfried-Claren-Straße 26, W-5300 Bonn 1, \\ Federal Republic of Germany
}

Received October 29, 1991; revised form January 10, 1992

\begin{abstract}
We show that two natural approaches to quantum gravity coincide. This identity is nontrivial and relies on the equivalence of each approach to $\mathrm{KdV}$ equations. We also investigate related mathematical problems.
\end{abstract}

\section{Witten's Conjecture}

1.1. Two-Dimensional Gravity(ies). Quantum gravity, although not well-defined, looks like integration over the (infinite-dimensional) space of riemannian metrics on manifolds modulo diffeomorphisms. There are at least two mathematically consistent approaches to two-dimensional gravity.

The first one was developed by [KB, DS, GM] and can be called "enumeration of triangulations." Any triangulation of the surface determines some singular metric obtained from the arrangement of equilateral triangles. One can imagine that when the number of triangles tends to infinity these singular metrics approximate "random metrics" on surfaces. Thus we are led to the problem of finding the asymptotics of the number of triangulations of surfaces of fixed genus into the given growing number of triangles. It was shown (using Feynman diagram techniques) that this problem together with some modifications is equivalent to describing the asymptotic behaviour of the integrals $\int \exp (\operatorname{tr} P(X)) d X$, where $X$ runs over the space of hermitian $N \times N$-matrices, $N \rightarrow \infty$ and $P$ is a polynomial depending (in some way) on $N$. These integrals were evaluated using orthogonal polynomials. It turns out that discrete Toda lattice equations hold. In the limit the Korteweg-de Vries equation arises. The partition function of the two-dimensional gravity for this approach is a series in an infinite number of variables and coincides with the logarithm of some $\tau$-function for KdV-hierarchy.

Another approach is to choose some specific action. Using supersymmetry the integral over the space of all metrics reduces to the integral over the finitedimensional space of conformal structures. The last integral has a cohomological description as an intersection theory on the compactified moduli space of

* Accepted as doctoral dissertation by the Mathematisch-Naturwissenschaftliche Fakultät, University of Bonn, FRG 
complex curves (see the next subsection for precise definitions). Again some series in an infinite number of variables arises. All number related to surfaces of genus less than or equal to 3 were computed using algebraic geometry.

E. Witten conjectured [W1] that the partition functions for both approaches coincide. The reason for this conjecture is an irrational (for mathematicians) idea, that gravity is unique.

Our way to compute the partition function for the second approach (and thus to check Witten's conjecture) uses Feynman diagram techniques and matrix integrals but in another way. Our matrix integral does not look like the standard matrix integral from the first variant of gravity. The coincidence of the two integrals is a nontrivial identity and was proven (in several ways) using the equivalence of noth integrals to $\mathrm{KdV}$ equations.

1.2. Notations. Let $g$ and $n$ be integers satisfying the conditions

$$
g \geqq 0, \quad n>0, \quad 2-2 g-n<0 .
$$

Denote by $\mathscr{M}_{g, n}$ the moduli orbispace (for this notion see Appendix A) of smooth complete complex curves of genus $g$ with $n$ distinct marked points $x_{1}, \ldots, x_{n}$, and $\overline{\mathscr{M}}_{g, n}$ the Deligne-Mumford smooth compactification (see [M], footnote on page 285). It is the moduli orbispace of complete connected curves $C$ with $n$ distinct marked points $x_{i}$ satisfying the following conditions:

(1) all singularities of $C$ are ordinary double points,

(2) the marked points are smooth,

(3) the Euler characteristic of any connected component of $C \backslash(\operatorname{Sing}(C) \cup$ $\left.\left\{x_{1}, \ldots, x_{n}\right\}\right)$ is negative and the sum of these numbers is equal to $2-2 g-n$.

Both spaces $\mathscr{M}_{g, n}$ and $\overline{\mathscr{M}}_{g, n}$ will be endowed with the usual (Hausdorff) topology of an analytic space.

Let $\mathscr{L}_{i}, i=1, \ldots, n$ be line bundles on $\overline{\mathscr{M}}_{g, n}$. The fiber of $\mathscr{L}_{i}$ at $(C$; $\left.x_{1}, \ldots, x_{n}\right)$ is the cotangent space $T_{x_{i}}^{*} C$.

Introduce the infinite sequence of indeterminates $\tau_{0}, \tau_{1}, \ldots$ Let $d_{1}, \ldots, d_{n}$ be non-negative integers satisfying

$$
\sum_{i=1}^{n} d_{i}=\operatorname{dim}_{\mathbf{C}} \overline{\mathscr{M}}_{g, n}=3 g-3+n .
$$

Denote by $\left\langle\tau_{d_{1}} \ldots \tau_{d_{n}}\right\rangle$ the intersection index

$$
\int_{\tilde{\mathscr{M}}_{g, n}} \prod_{i=1}^{n} c_{1}\left(\mathscr{L}_{i}\right)^{d_{i}}
$$

For example, $\left\langle\tau_{0} \tau_{0} \tau_{0}\right\rangle=1,\left\langle\tau_{1}\right\rangle=\frac{1}{24}$ (see [W1]). By Arakelov's theorem all the numbers $\left\langle\tau_{d_{1}} \ldots \tau_{d_{n}}\right\rangle$ are non-negative ([M]).

We set $\left\langle\tau_{d_{1}} \ldots \tau_{d_{n}}\right\rangle$ equal to zero if the "genus" $g$, defined by the formula

$$
g=\frac{1}{3}\left(\sum_{i=1}^{n} d_{i}-n+3\right),
$$

is not an integer or if $n=0$. In this way we have defined a linear functional

$$
\langle\cdots\rangle: \mathbf{Q}\left[\tau_{0}, \tau_{1}, \ldots\right] \rightarrow \mathbf{Q} .
$$


The generating function proposed by E. Witten is a formal series in another sequence of indeterminates $t_{0}, t_{1}, \ldots$ :

$$
F\left(t_{0}, t_{1}, \ldots\right)=\left\langle\exp \left(\sum_{i=0}^{\infty} t_{i} \tau_{i}\right)\right\rangle=\sum_{(k)}\left\langle\tau_{0}^{k_{0}} \tau_{1}^{k_{1}} \cdots\right\rangle \prod_{i=0}^{\infty} \frac{t_{i}^{k_{i}}}{k_{i} !} .
$$

Witten's conjecture asserts that the series $F$ coincides with the partition function in the standard matrix model theory and, in particular, obeys the Korteweg-de Vries hierarchy, the first equation of which is the classical KdV equation

$$
\frac{\partial U}{\partial t_{1}}=U \frac{\partial U}{\partial t_{0}}+\frac{1}{12} \frac{\partial^{3} U}{\partial t_{0}^{3}} \quad\left(U=\frac{\partial^{2} F}{\partial t_{0}^{2}}\right) .
$$
4.3).

Recently E. Witten proposed a generalization of this conjecture (see Sect.

1.3. Statements. Let $\Lambda$ be a positive definite hermitian $N \times N$ matrix. Denote by $d \mu_{\Lambda}(X)$ the probability measure on the vector space of hermitian $N \times N$ matrices given by the density

$$
c_{\Lambda} \exp \left(-\frac{\operatorname{tr} X^{2} \Lambda}{2}\right) d X, \quad c_{\Lambda}=\operatorname{det}\left(\frac{1}{4 \pi}(\Lambda \otimes 1+1 \otimes \Lambda)\right)^{1 / 2} .
$$

The constant $c_{\Lambda}$ is chosen so that the condition $\int d \mu_{\Lambda}(X)=1$ is satisfied.

Define functions $t_{i}, i=0,1, \ldots$ of the matrix $\Lambda$ :

$$
t_{i}(\Lambda)=-(2 i-1) ! ! \operatorname{tr} \Lambda^{-(2 i+1)},
$$

where $(2 i-1) ! !=1 \cdot 3 \cdot \ldots \cdot(2 i-1)$.

Theorem 1.1. The formal series $F\left(t_{0}(\Lambda), t_{1}(\Lambda), \ldots\right)$ is an asymptotic expansion of

when $\Lambda^{-1} \rightarrow 0$.

$$
\log \left(\int \exp \left(\frac{\sqrt{-1}}{6} \operatorname{tr} X^{3}\right) d \mu_{\Lambda}(X)\right)
$$

The proof of this theorem is contained in Sect. 3.2. For any fixed size of matrix $\Lambda$ the functions $t_{i}(\Lambda)$, for $i=0, \ldots, N-1$ are algebraically independent. So, to obtain from Theorem 1.1 the terms in the series $F$ up to any fixed order, we have to take the integral over the space of matrices of sufficiently large size.

Using Theorem 1.1 we prove

Theorem 1.2. The series $\exp (F)$ in variables $T_{2 i+1}:=t_{i} /(2 i+1)$ !! is a $\tau$-function for the $\mathrm{KdV}$-hierarchy.

It follows from Theorem 1.2 that Witten's conjecture is true. At the moment we know at least 3 different proofs of Theorem 1.2. The shortest proof is contained in Sects. 4.1 and 4.2. Other proofs (see [K2, W2]) are more complicated.

One can easily deduce from Theorem 1.1 that for any $n \geqq 0$ the integral

$$
\int\left(\operatorname{tr} X^{3}\right)^{n} d \mu_{\Lambda}(X)
$$

is a polynomial in variables $t_{i}$. The following theorem generalizes this fact: 
Theorem 1.3. There exists a linear map

$$
I: \mathbf{Q}\left[x_{1}, x_{3}, x_{5}, \ldots\right] \rightarrow \mathbf{Q}\left[l_{1}, l_{3}, l_{5}, \ldots\right]
$$

such that for any positive definite hermitian matrix $\Lambda$ and for any $P \in \mathbf{Q}\left[x_{1}, x_{3}, \ldots\right]$ one has

$$
\int P\left(\operatorname{tr} X, \operatorname{tr} X^{3}, \ldots\right) d \mu_{\Lambda}(X)=I(P)\left(\operatorname{tr} \Lambda^{-1}, \operatorname{tr} \Lambda^{-3}, \ldots\right) .
$$

This theorem is proven in Sect. 3.3. Some conjectures concerning $I$ are presented in Sect. 3.4.

\section{Reduction to the "Combinatorial" Problem}

2.1. Strebel Differentials and Ribbon Graphs. In this section we will describe an equivalence, due to R. Penner, J. Harer, D. Mumford and W. Thurston between the "decorated" moduli space of algebraic curves and the moduli space of ribbon graphs (see Th. 2.2). We choose here the version of this equivalence based on conformal geometry and results of K. Strebel.

A quadratic differential $\varphi$ on a Riemann surface $C$ of finite type is a holomorphic section of the line bundle $\left(T^{*}\right)^{\otimes 2}$. A nonzero quadratic differential defines a flat metric on the complement of the discrete set of its zeroes according to a formula in a local coordinate $z$ :

$$
|\varphi(z)| \cdot|d z|^{2} \text {, where } \varphi=\varphi(z) d z^{2} .
$$

A horizontal trajectory of a quadratic differential is a curve along which $\varphi(z) d z^{2}$ is real and positive. Jenkins-Strebel quadratic differentials are those for which the union of nonclosed trajectories has measure zero.

Nonclosed trajectories of a JS differential decompose the surface into the maximal ring domains swept out by closed trajectories. These ring domains can be annuli or punctured disks. All trajectories from any fixed maximal ring domain have the same length, the circumference of domain. In late 60's $\mathrm{K}$. Strebel proved the following theorem:

Theorem 2.1. For any connected Riemann surface $C$ and $n$ distinct points $x_{1}, \ldots, x_{n} \in C, n>0, n>\chi(C)$ and $n$ positive real numbers $p_{1}, \ldots, p_{n}$ there exists a unique JS quadratic differential on $C \backslash\left\{x_{1}, \ldots, x_{n}\right\}$ whose maximal ring domains are $n$ punctured disks $D_{i}$ surrounding points $x_{i}$ with circumference $p_{i}$.

This theorem is essentially Theorem 23.2 (for $n=1$ ) and Theorem 23.5 (for $n \geqq 2$ ) in [S]. The reader can also see a recent exposition of Strebel's theory in $[Z]$. In this section we consider only compact surfaces.

The union of all nonclosed trajectories and zeroes of a JS differential $\varphi$ is a finite graph (=1-dimensional $C W$-complex) $\Gamma_{\varphi}$ embedded in the surface. A vertex of $\Gamma_{\varphi}$ which is a zero of $\varphi$ of $k^{\text {th }}$ order has valency $k+2 \geqq 3$. The complement to $\Gamma_{\varphi}$ consists of open disks, hence we obtain a cell decomposition of $C$. The graph $\Gamma_{\varphi}$ carries two additional structures

(1) for each vertex a cyclic order on the set of germs of edges meeting this vertex is fixed, (we say that $\Gamma_{\varphi}$ is a ribbon graph),

(2) to each edge is attached a positive real number, its length (a metric on the graph). 

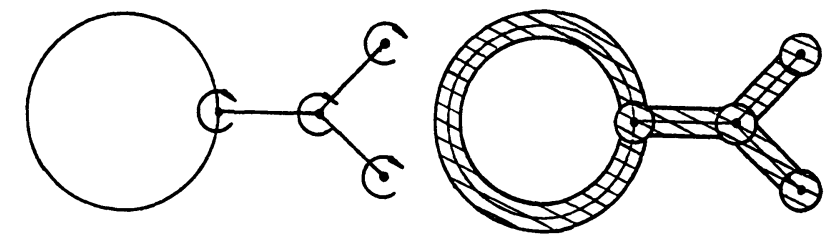

Fig. 1

$$
\text { graph }
$$

surface
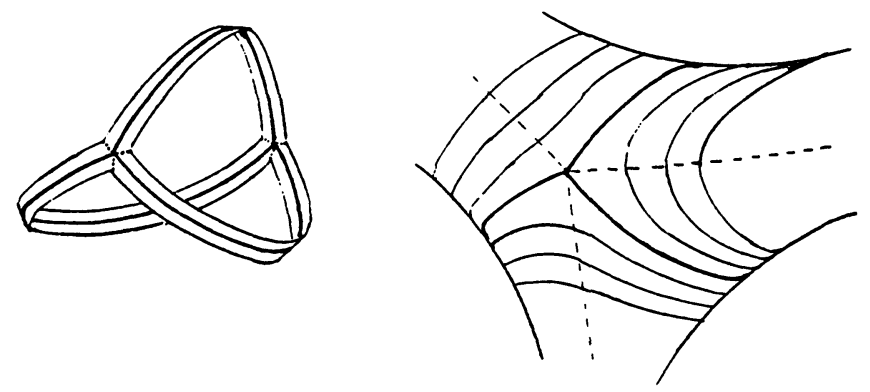

Fig. 2

In the reverse direction, for any (connected) finite ribbon graph $\Gamma$ with metric whose valency at each vertex is greater than or equal to 3 we can construct JS differential.

The first step is to replace vertices by small disks, replace edges by oriented open ribbons and glue them at each vertex according to the cyclic order chosen for this vertex (see Fig. 1). In this way we obtain an oriented noncompact surface on which the graph is drawn. We can divide this surface into rectangles, where each rectangle is homeomorphic to the product of the corresponding edge of the graph by $[0,+\infty)$. Endow each rectangle by the standard flat metrics of the semistrip with width equal to the length of the corresponding edge of $\Gamma$. After isometrically gluing together all these rectangles we obtain a surface with a flat metric defined almost everywhere. The surface is glued from a finite number of infinite tubes (see Fig. 2). It is easy to see that there exists a unique complex structure on this surface compatible with the metric. This surface is a compact Riemann surface $C$ minus a nonempty subset $\left\{x_{1}, \ldots, x_{n}\right\} \subset C$. There exists a unique quadratic differential on $C$ whose trajectories restricted to the semistrips are the standard vertical intervals.

Hence we have proved the following result:

Theorem-Notation 2.2. Let $\mathscr{M}_{g, n}^{\text {comb }}$ denote the set of equivalence classes of connected ribbon graphs with metric and with valency of each vertex greater than or equal to 3 such that the corresponding noncompact surface has genus $g$ and $n$ holes numbered by $1, \ldots, n$ (numbered graphs). The map $\mathscr{M}_{g, n} \times \mathbf{R}_{+}^{n} \rightarrow \mathscr{M}_{g, n}^{\text {comb }}$ which associated to the surface $C$ and numbers $p_{1}, \ldots, p_{n}$ the critical graph of the canonical JS-differential (from Theorem 2.1) is one-to-one. 
We can endow the spaces $\mathscr{M}_{g, n}^{\text {comb }}$ with some natural topology and orbispace structure (see Appendix B). The isomorphism from Theorem 2.2 is an isomorphism of orbispaces.

The combinatorial type of the underlying ribbon graphs defines a stratification on $\mathscr{M}_{g, n}^{\text {comb }}$ with the dimension of a stratum equal to the number of edges. The open strata corresponds to the 3-valent graphs and have dimension equal to $6 g-6+3 n$.

Remarks. Probably D. Mumford was the first who noticed that the stratification of the moduli space arises from Strebel theorems, see [H]. R. Penner [P1] described a stratification of $\mathscr{M}_{g, n} \times \mathbf{R}_{+}^{n}$ using hyperbolic geometry. In his picture simplicial coordinates on strata arose and lead to another homeomorphism $\mathscr{M}_{g, n} \times \mathbf{R}_{+}^{n} \rightarrow \mathscr{M}_{g, n}^{\text {comb }}$.

Both stratifications are combinatorially equivalent, but geometrically they are different. R. Penner uses the name "fatgraph" for ribbon graphs. He calls "the decorated Teichmüller space" the universal cover of $\mathscr{M}_{g, n} \times \mathbf{R}_{+}^{n}$.

Example. $g=0, n=3$. Recall that $\mathscr{M}_{0,3}$ is the one point set. There are 7 numbered graphs (see Fig. 3). $\mathscr{M}_{g, n}^{\text {comb }}$ consists in 3 copies of $\mathbf{R}_{+}^{2}$ and 4 copies of $\mathbf{R}_{+}^{3}$. The map $\mathscr{M}_{g, n}^{\text {comb }} \rightarrow \mathbf{R}_{+}^{3}$ given by the triple $\left(p_{1}, p_{2}, p_{3}\right)$ of perimeters of tubes is a homeomorphism. The "central" 3-dimensional stratum corresponds to the triples satisfying strict triangle inequalities.

Notations. For a ribbon graph $\Gamma$, denote by $X\left(=X_{\Gamma}\right)$ the set of edges of the graph together with a choice of orientation. Let $s_{0}$ and $s_{1}$ be two permutations of $X: s_{1}$ is the operation of changing orientation and $s_{0}$ permutes cyclically all oriented edges with a common source. The set $X_{0}=X /\left\langle s_{0}\right\rangle$ is canonically equivalent to the set of vertices of $\Gamma$ and the set $X_{1}=X / s_{1}$ is equivalent to the set of edges. Denote by $s_{2}$ the permutation $s_{0}^{-1} s_{1}$. The set $X_{2}=X \mid\left\langle s_{2}\right\rangle$ is equivalent to the set of 2-cells of the cell-decomposition associated with $\Gamma$ (see Fig. 4). Later we will use the following notation: $[x]_{i}, i=0,1,2$ is the image of $x \in X$ under the projection map $X \rightarrow X_{i}$. The length of an edge $e \in X_{1}$ is denoted by $l(e)$.

It is easy to see that there is an equivalence between

(1) ribbon graphs without isolated vertices,

(2) triples $\left(X, s_{0}, s_{1}\right)$ where $X$ is a finite set, $s_{0}, s_{1} \in \operatorname{Aut}(X), s_{1}$ is a free involution,

(3) cell decompositions of closed oriented surfaces which have no components of the type $S^{2}=D^{0} \cup D^{2}$.

2.2. Polygon Bundles. For each integer $N$ let us denote by $B U(1) \leqq N$ comb the set of equivalence classes of all sequences of positive real numbers $l_{1}, \ldots, l_{k}$, $1 \leqq k \leqq N$, modulo cyclic permutations. This set carries a natural topology: when $\bar{l}_{i} \rightarrow 0$ for some $i$ then the limit is obtained by removing the $i^{\text {th }}$ term. Each sequence has as automorphism group a finite cyclic group. This provides $B U(1) \stackrel{\text { comb }}{\subseteq}$ with an orbispace structure. Define $B U(1)^{\text {comb }}$ to be the direct limit of $B U(1) \leqq N$ over all $N$.

In other words $B U(1)^{\text {comb }}$ is the moduli (orbi) space of numbered ribbon graphs with metric whose underlying graphs are homeomorphic to the circle. There is an $S^{1}$-bundle over this orbispace whose total space $E U(1)^{\text {comb }}$ is an 


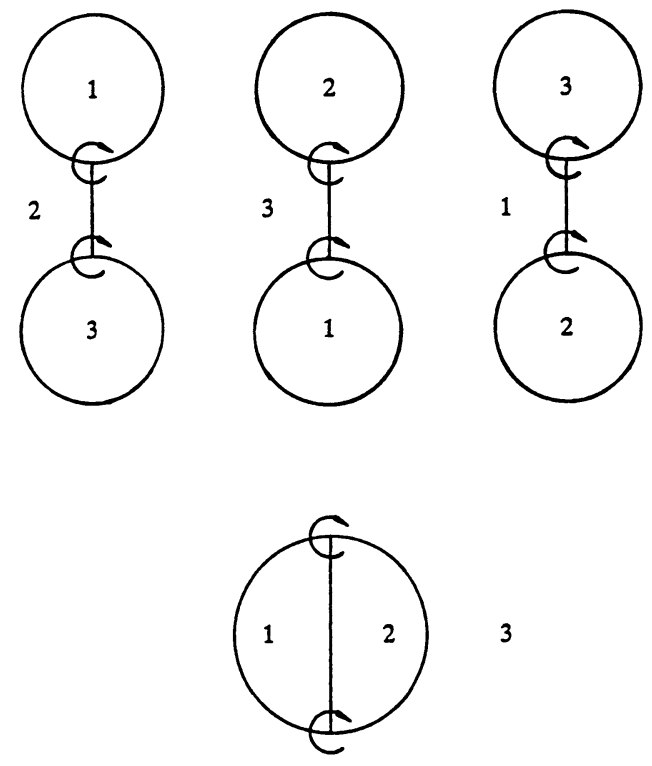

Fig. 3
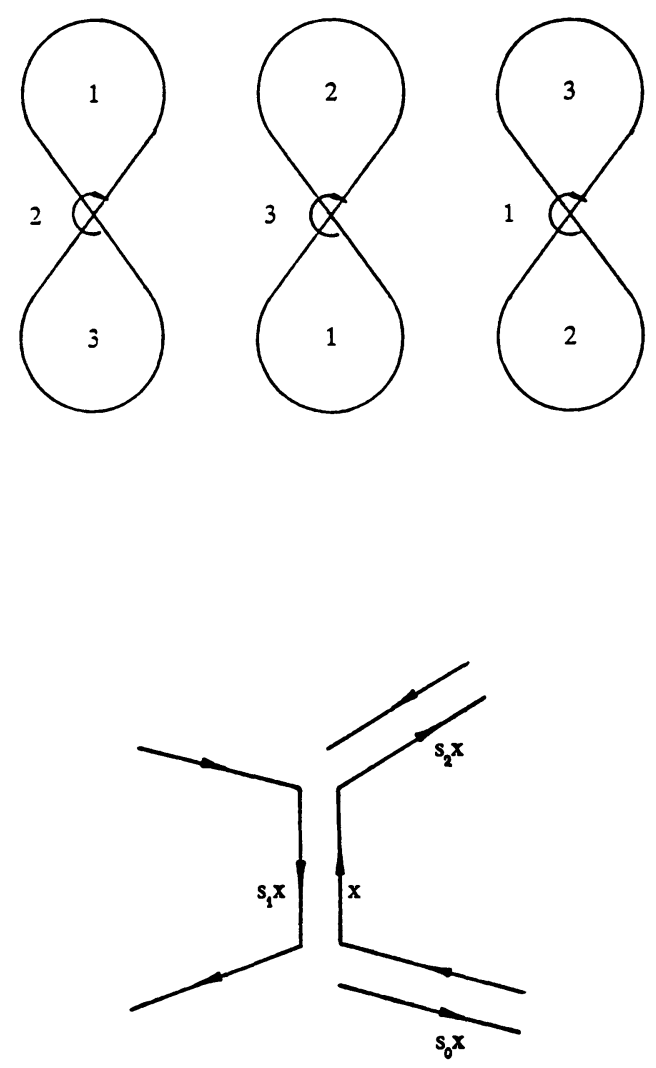

Fig. 4 
ordinary space. One can check that the space $E U(1)^{\text {comb }}$ is contractible. This fact explains our notations. The fiber of the bundle over the equivalence class of sequences $l_{1}, \ldots, l_{k}$ is a union of intervals of lengths $l_{1}, \ldots, l_{k}$ with pairwise glued ends, i.e. a polygon.

The moduli space of ribbon graphs with metric $\mathscr{M}_{g, n}^{\text {comb }}$ maps in an obvious way to $B U(1)^{\text {comb }}$ (if we fix $i, 1 \leqq i \leqq n$ ): the $i^{\text {th }}$ boundary component of a ribbon graph with metric is a polygon.

Theorem 2.3. The map $\mathscr{M}_{g, n} \times \mathbf{R}_{+}^{n} \rightarrow\left(B U(1)^{\mathrm{comb}}\right)^{n}$, which is the composition of the isomorphism $\mathscr{M}_{g, n} \times \mathbf{R}_{+}^{n} \cong \mathscr{M}_{g, n}^{\text {comb }}$ and maps to $B U(1)^{\text {comb }}$ described above, extends continuously to $\overline{\mathscr{M}}_{g, n} \times \mathbf{R}_{+}^{n}$. The inverse images of $S^{1}$-bundles are naturally isomorphic to the circle bundles associated with the complex line bundles $\mathscr{L}_{i}$.

The proof of this theorem is quite technical (see some information in Appendix B).

Let us now compute the first Chern class of the circle bundle on $B U(1)^{\text {comb }}$. The points of $E U(1)^{\text {comb }}$ can be identified with pairs $(p, S)$ where $p$ is a positive real number and $S$ is a nonempty finite subset of the circle $\mathbf{R} / p \mathbf{Z}$. Here $p$ is the perimeter of the polygon, edges of the polygon are connected components of $\mathbf{R} / p \mathbf{Z} \backslash S$. Denote by $0 \leqq \phi_{1}<\cdots<\phi_{k}<p$ representatives of points of $S$. The lengths of the edges of the polygon are

$$
l_{i}=\phi_{i+1}-\phi_{i} \quad(i=1, \ldots, k-1), l_{k}=p+\phi_{1}-\phi_{k} .
$$

Denote by $\alpha$ the 1 -form on $E U(1)^{\text {comb }}$ equal to

$$
\sum_{i=1}^{k} l_{i} / p \times d\left(\phi_{i} / p\right)
$$

It is easy to check that $\alpha$ is well-defined and that the integral of $\alpha$ over each fiber of the universal bundle $E U(1)^{\text {comb }} \rightarrow B U(1)^{\text {comb }}$ is equal to -1 . The differential $d \alpha$ is the pullback of a 2 -form $\omega$ on the base $B U(1)^{\text {comb }}$,

$$
\omega=\sum_{1 \leqq i \leqq j \leqq k-1} d\left(l_{i} / p\right) \wedge d\left(l_{j} / p\right)
$$

So, $\omega$ is obtained by transgression from $\alpha$ and we have proved the following result:

Lemma 2.1. The pullback $\omega_{i}$ of the form $\omega$ under the $i^{\text {th }}$ map $\overline{\mathscr{M}}_{g, n} \times \mathbf{R}_{+}^{n} \rightarrow$ $B U(1)^{\text {comb }}$ represents the class $c_{1}\left(\mathscr{L}_{i}\right)$.

Denote by $\pi: \mathscr{M}_{g, n}^{\text {comb }} \rightarrow \mathbf{R}_{+}^{n}$ the projection given by the sequence of perimeters of tubes. We have the following formula for intersection numbers:

$$
\left\langle\tau_{d_{1}} \ldots \tau_{d_{n}}\right\rangle=\int_{\pi^{-1}\left(p_{*}\right)} \prod_{i=1}^{n} \omega_{i}^{d_{i}}
$$

where $p_{*}=\left(p_{1}, \ldots, p_{n}\right)$ is an arbitrary sequence of positive real numbers. The only problem with this formula is to describe the orientation of open strata in $\mathscr{M}_{g, n}^{\text {comb }}$ arising from the complex structure. 


\section{Matrix Integrals}

3.1. Main Identity. Denote by $\Omega$ the two-form on open strata of $\mathscr{M}_{g, n}^{\text {comb }}$ equal to the sum $\sum p_{i}^{2} \omega_{i}$. The reason for this choice is explained by the obvious

Lemma 3.1. The restriction of the form $\Omega$ to the fibers of $\pi$ has constant coefficients in the coordinates $(l(e)), e \in X_{1}$.

One can check that $\Omega$ is nondegenerate along the fibers of $\pi$ and defines some orientation compatible along the codimension one strata (see Lemma 3.2 in Sect. 3.3 and Lemma C.1 in Appendix C).

Denote by $d$ the complex dimension of $\mathscr{M}_{g, n}, d=3 g-3+n$. The volume of the fiber of $\pi$ with respect to $\Omega$ is

$$
\begin{aligned}
\operatorname{vol}\left(\pi^{-1}\left(p_{1}, \ldots, p_{n}\right)\right) & =\int_{\pi^{-1}\left(p_{*}\right)} \frac{\Omega^{d}}{d !}=\frac{1}{d !} \int_{\pi^{-1}\left(p_{*}\right)}\left(p_{1}^{2} c_{1}\left(\mathscr{L}_{1}\right)+\cdots+p_{n}^{2} c_{1}\left(\mathscr{L}_{n}\right)\right)^{d} \\
& =\operatorname{sgn} \times \sum_{d_{*}: \Sigma d_{i}=d} \prod_{i=1}^{n} \frac{p_{i}^{2 d_{i}}}{d_{i} !} \times\left\langle\tau_{d_{1}} \ldots \tau_{d_{n}}\right\rangle .
\end{aligned}
$$

The symbol sgn equals \pm 1 and denotes the ratio of the orientation coming from the complex structure and the orientation cominmg from the symplectic structure. Recall that $\left\langle\tau_{d_{1}} \ldots \tau_{d_{n}}\right\rangle \geqq 0$, so by the positivity of the volume $\operatorname{sgn}=+1$.

Let $\lambda_{i}, i=1, \ldots, n$ be real positive numbers. The Laplace transform of volumes of fibers of $\pi$ is

$$
\begin{gathered}
\int_{0}^{\infty} \cdots \int_{0}^{\infty} \prod_{i=1}^{n} d p_{i} \times \exp \left(-\sum \lambda_{i} p_{i}\right) \times \operatorname{vol}\left(\pi^{-1}\left(p_{*}\right)\right) \\
\quad=\sum_{d_{*}: \Sigma d_{i}=d}\left\langle\tau_{d_{1}} \ldots \tau_{d_{n}}\right\rangle \prod_{i=1}^{n} \frac{1}{d_{i} !} \int_{0}^{+\infty} p_{i}^{2 d_{i}} e^{-\lambda_{i} p_{i}} d p_{i} \\
\quad=\sum_{d_{*}: \Sigma d_{i}=d}\left\langle\tau_{d_{1}} \ldots \tau_{d_{n}}\right\rangle \prod_{i=1}^{n} \frac{\left(2 d_{i}\right) !}{d_{i} !} \lambda_{i}^{-\left(2 d_{i}+1\right)} .
\end{gathered}
$$

Let us write the left-hand side of this equality in the following form:

$$
\text { L.H.S. }=\underset{\mathcal{M}_{g, n}^{c o m b}}{\int_{m}} \varrho \times \exp \left(-\sum \lambda_{i} p_{i}\right) \times \prod_{e \in X_{1}}|d l(e)|,
$$

where $\varrho$ is a positive function defined on open cells, $\varrho$ is equal to the ratio of measures:

$$
\varrho=\left(\prod_{i=1}^{n}\left|d p_{i}\right| \times \frac{\Omega^{d}}{d !}\right): \prod_{e \in X_{1}}|d l(e)| .
$$

From Lemma 3.1 it follows that $\varrho$ is locally constant and depends only on the combinatorial type of a 3-valent graph. It is shown in Appendix $C$ that

$$
\varrho=2^{2 n+5 g-5}=4^{d} 2^{1-g}=2^{d+\# X_{1}-\# X_{0}} .
$$

The integral

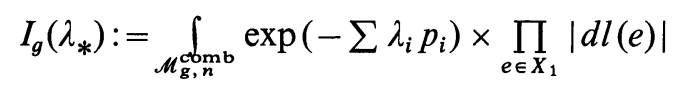

is equal to the sum of integrals over all open strata in $\mathscr{M}_{g, n}^{\text {comb }}$. 
For an edge $e=[x]_{1} \in X_{1}$ denote by $\tilde{\lambda}(e)$ the sum $\lambda\left([x]_{2}\right)+\lambda\left(\left[s_{1} x\right]_{2}\right)$, where $\lambda$ is considered as a function $\lambda: x_{2} \rightarrow \mathbf{R}_{+}$. For any ribbon graph

$$
\exp \left(-\sum \lambda_{i} p_{i}\right)=\exp \left(-\sum_{x \in X} l\left([x]_{1}\right) \lambda\left([x]_{2}\right)\right)=\prod_{e \in X_{1}} \exp (-l(e) \tilde{\lambda}(e)) .
$$

Let us denote by $G_{g, n}$ the set of equivalence classes of 3-valent graphs with numbering $X_{2} \cong\{1, \ldots, n\}$ from $\mathscr{M}_{g, n}^{\text {comb }}$. Now we can compute the integral:

$$
\begin{aligned}
I_{g}\left(\lambda_{*}\right) & =\sum_{\Gamma \in \mathbf{G}_{\boldsymbol{g}, n}} \frac{1}{\# \operatorname{Aut} \Gamma} \prod_{e \in X_{1}} \int_{0}^{+\infty} \exp (-l(e) \tilde{\lambda}(e)) d l(e) \\
& =\sum_{\Gamma \in \mathbf{G}_{\boldsymbol{g}, n}} \frac{1}{\# \operatorname{Aut} \Gamma} \prod_{e \in X_{1}} \frac{1}{\tilde{\lambda}(e)} .
\end{aligned}
$$

After multiplication by an appropriate power of 2 we obtain the main identity:

$$
\sum_{d_{*}: \Sigma d_{i}=d}\left\langle\tau_{d_{1}} \ldots \tau_{d_{n}}\right\rangle \prod_{i=1}^{n} \frac{\left(2 d_{i}-1\right) ! !}{\lambda_{i}^{2 d_{i}+1}}=\sum_{\Gamma \in G_{g, n}} \frac{2^{-\# X_{0}}}{\# \operatorname{Aut} \Gamma} \prod_{e \in X_{1}} \frac{2}{\tilde{\lambda}(e)}
$$

Example. $g=0, n=3 . G_{0,3}$ contains 4 graphs, all of which have no nontrivial automorphisms (see Fig. 3). The main identity for this case is

$$
\begin{aligned}
\frac{2}{2 \lambda_{1}\left(\lambda_{1}+\lambda_{2}\right)\left(\lambda_{1}+\lambda_{3}\right)} & +\frac{2}{2 \lambda_{2}\left(\lambda_{2}+\lambda_{3}\right)\left(\lambda_{2}+\lambda_{1}\right)}+\frac{2}{2 \lambda_{3}\left(\lambda_{3}+\lambda_{1}\right)\left(\lambda_{3}+\lambda_{2}\right)} \\
& +\frac{2}{\left(\lambda_{1}+\lambda_{2}\right)\left(\lambda_{2}+\lambda_{3}\right)\left(\lambda_{3}+\lambda_{1}\right)}=\frac{1}{\lambda_{1} \lambda_{2} \lambda_{3}}=\frac{\left\langle\tau_{0} \tau_{0} \tau_{0}\right\rangle}{\lambda_{1} \lambda_{2} \lambda_{3}} .
\end{aligned}
$$

3.2. Matrix Model (the Proof of Theorem 1.1). Let $\Lambda=\operatorname{diag}\left(\Lambda_{1}, \ldots, \Lambda_{N}\right)$ be a positive diagonal hermitian matrix. Denote (as in Sect. 1.3) by $t_{i}(\Lambda)$ the expression

$$
-(2 i-1) ! ! \operatorname{tr} \Lambda^{-(2 i+1)}=-(2 i-1) ! ! \sum_{j=1}^{N} \Lambda_{j}^{-(2 i+1)}
$$

Let us take a formal sum over all $g, n$ of the main identity from the previous section:

$$
\begin{aligned}
& F\left(t_{0}(\Lambda), t_{1}(\Lambda), \ldots\right)=\sum_{n \geqq 1, d_{1}, \ldots, d_{n} \geqq 0}\left\langle\tau_{d_{1}} \ldots \tau_{d_{n}}\right\rangle \times \frac{1}{n !} \times t_{d_{1}}(\Lambda) \ldots t_{d_{n}}(\Lambda) \\
& \quad=\sum_{n \geqq 1, d_{1}, \ldots, d_{n} \geqq 0} \frac{(-1)^{n}}{n !}\left\langle\tau_{d_{1}} \ldots \tau_{d_{n}}\right\rangle \sum_{1 \leqq j_{1}, \ldots, j_{n} \leqq N} \prod_{i=1}^{n}\left(2 d_{i}-1\right) ! ! \Lambda_{j_{i}}^{-\left(2 d_{i}+1\right)} \\
& =\sum_{\Gamma \in G_{N}} \frac{(\sqrt{-1} / 2)^{\# X_{0}}}{\# \text { Aut } \Gamma} \times \prod_{e \in X_{1}} \frac{2}{\tilde{\lambda}(e)} .
\end{aligned}
$$

In the last term $G_{N}$ denotes the set of equivalence classes of connected nonempty 3 -valent ribbon graphs together with maps $c: X_{2} \rightarrow\{1, \ldots, N\}$ (colorings of $X_{2}$ in $N$ colors), $\tilde{\lambda}\left([x]_{1}\right)=\Lambda_{c\left([x]_{2}\right)}+\Lambda_{c\left(\left[s_{1} x\right]_{2}\right)}$. 

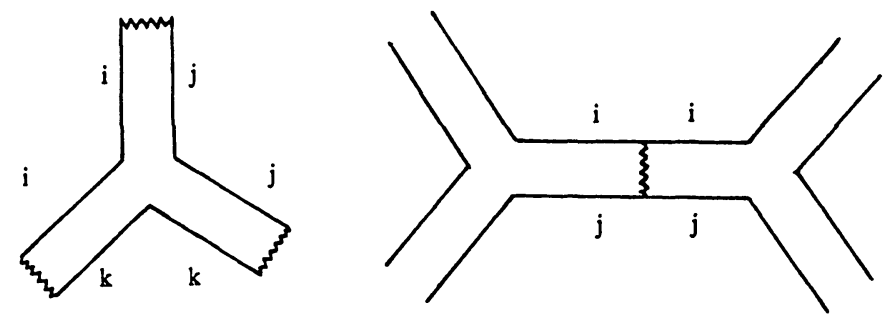

Fig. 5

Elements of $G_{N}$ are all connected nonempty graphs obtained by glueing special vertices (see Fig. 5) in such a way that corresponding indices on glued edges coincide. By Feynman diagram techniques (see [BIZ]) the last series is an asymptotic expansion for

$$
\log \left(\int \exp \left(\frac{\sqrt{-1}}{6} \operatorname{tr} X^{3}\right) d \mu_{(\Lambda)}(X)\right),
$$

where $d \mu_{(\Lambda)}(X)$ is a gaussian measure on the vector space of hermitian matrices such that

$$
\int X_{i j} X_{k l} d \mu_{(\Lambda)}(X)=\delta_{i l} \delta_{j k} \frac{2}{\Lambda_{i}+\Lambda_{j}}
$$

This is exactly the measure $d \mu_{\Lambda}(X)$ introduced in Sect. 1.3. Thus, Theorem 1.1 is proven.

Remark. The idea to apply Feynman diagram techniques and matrix integrals to the topology of the moduli space of curves appears for the first time in R. Penner's computation of the Euler characteristic of $\mathscr{M}_{g, n}([\mathrm{P} 2])$. The first computation of the same number by J. Harer and D. Zagier [HZ] also uses gaussian integrals over the space of hermitian matrices. Recently we found a simple argument which reduces the integral to the case of one variable and simplifies radically the analytic part of the computation (see Appendix D). There are now proofs also without matrix integration, see [IZ2].

3.3. The Proof of Theorem 1.3. Fix a positive number $n$ and a sequence of nonnegative integers $m_{0}, m_{1}, \ldots, m_{i}=0$ for all sufficiently large $i$. Denote by $\mathscr{M}_{m_{*}, n}$ the moduli orbispace of connected numbered ribbon graphs $\Gamma$ with metric, such that \# $X_{2}=n$ and $\Gamma$ have $m_{i}$ vertices of valency $2 i+1$ and no vertices of even valencies. If $m_{i}=0$ for $i \neq 1$ then $\mathscr{M}_{m_{*}, n}$ is the union of all open strata of $\mathscr{M}_{g, n}^{\text {comb }}$ for some $g$. The form $\Omega$ from Sect. 3.1 defines a symplectic structure on fibers of the map $\pi: \mathscr{M}_{m_{*}, n} \rightarrow \mathbf{R}_{+}^{n}$. The volume form $\exp (\Omega) \wedge d p_{1} \wedge \cdots \wedge d p_{n}$ defines an orientation on all components of $\mathscr{M}_{m_{*}, n}$. I will leave without proof the following simple lemma.

Lemma 3.2. The map $\mathscr{M}_{m_{*}, n} \rightarrow\left(B U(1)^{\text {comb }}\right)^{n}$ from the orbifold $\mathscr{M}_{m_{*}, n}$ endowed with the orientation described above defines a cycle with closed support on $\left(B U(1)^{\text {comb }}\right)^{n}$. 
One can integrate over this cycle any cohomology class with compact support on $(B U(1) \stackrel{\text { comb }}{n})^{n}$ for sufficiently large $N$.

Denote by $\left\langle\tau_{d_{1}} \ldots \tau_{d_{n}}\right\rangle_{m_{0}, m_{1}}, \ldots$ the rational number

$$
\int_{m_{*}, n} \prod_{i=1}^{n} \omega_{i}^{d_{i}} \times\left[\mathbf{R}_{+}^{n}\right]
$$

where $\left[\mathbf{R}_{+}^{n}\right] \in H_{\text {comp }}^{n}\left(\mathbf{R}_{+}^{n}\right)$ is the fundamental class with compact support.

We can repeat the computations from Sect. 3.1 using the formula (Appendix C)

$$
\varrho=4^{d} 2^{1-g}, \quad d:=\frac{1}{2}\left(\operatorname{dim} \mathscr{M}_{m_{*}, n}-n\right), \quad g:=\text { genus of } \Gamma \text { for any } \Gamma \in \mathscr{M}_{m_{*}, n} .
$$

One can introduce a series in an infinite number of variables of two types:

$$
Z\left(t_{0}, t_{1}, \ldots ; s_{0}, s_{1}, \ldots\right)=\exp \left(\sum_{n_{*}, m_{*}}\left\langle\tau_{d_{1}} \ldots \tau_{d_{n}}\right\rangle_{m_{0}, m_{1}}, \ldots \prod_{i=0}^{\infty} \frac{t_{i}^{n_{i}}}{n_{i} !} \prod_{j=0}^{\infty} s_{j}^{m_{j}}\right) .
$$

The same arguments as in Sect. 3.1 show that $Z\left(t_{*}(\Lambda), s_{*}\right)$ is an asymptotic expansion of

$$
\int \exp \left(\sqrt{-1} \sum_{j=0}^{\infty}(-1 / 2)^{j} s_{j} \frac{\operatorname{tr} X^{2 j+1}}{2 j+1}\right) d \mu_{\Lambda}(X)
$$

where $\Lambda^{-1} \rightarrow 0$ and $s_{*}$ is a fixed sequence of real numbers, $s_{j}=0$ for almost all $j$.

Consider $Z\left(t_{*}, s_{*}\right)$ as a series in indeterminates $s_{*}$. Its coefficients are polynomials in $t$. This gives a proof of Theorem 1.3.

3.4. Some Conjectures. We start from two "dual" conjectures concerning the series $Z\left(t_{*}, s_{*}\right)$ from the previous section. We are not fully confident in these conjectures.

Conjecture 3.1. $Z\left(t_{*}, s_{*}\right)$ is a $\tau$-function for $\mathrm{KdV}$-hierarchy in variables $T_{2 i+1}:=$ $t_{i} /(2 i+1)$ !! for arbitrary $s_{*}$.

Conjecture 3.2. $Z\left(t_{*}, s_{*}\right)$ is a $\tau$-function for $\mathrm{KdV}$-hierarchy in variables $T_{2 i+1}:=$ $s_{i} /(2 i+1)$ for arbitrary $t_{*}$.

A new conjecture of E. Witten (see [W2]) can also be formulated using series $Z$ :

Conjecture 3.3. There exists a linear isomorphism $\tilde{I}: \mathbf{Q}\left[\frac{\partial}{\partial t_{0}}, \frac{\partial}{\partial t_{1}}, \ldots\right] \cong$ $\mathbf{Q}\left[\frac{\partial}{\partial s_{0}}, \frac{\partial}{\partial s_{1}}, \ldots\right]$ such that $\forall P \in \mathbf{Q}\left[\frac{\partial}{\partial t_{0}}, \ldots\right], P(Z)=\tilde{I}(P)(Z)$ when $s_{1}=1$; $s_{j}=0$ for $j \neq 1$.

In other words, for any $d_{0}, d_{1}, \ldots$ there exists (?) a nonhomogeneous polynomial $P_{d_{*}}(X)=P_{d_{*}}\left(\operatorname{tr} X, \operatorname{tr} X^{3}, \ldots\right)$ of degree $\sum(2 i+1) d_{i}$ such that

$$
\frac{\partial^{d_{0}}}{\partial t_{0}^{d_{0}}} \frac{\partial^{d_{1}}}{\partial t_{1}^{d_{1}}} \ldots \exp (F)=\int P_{d_{*}}(X) \exp \left(\operatorname{tr} X^{3} / 6\right) d \mu_{\Lambda}(X)
$$


The last conjecture concerns cycles on $\overline{\mathscr{M}}_{q, n}$ arising in the proof of Theorem 1.3. If $m_{0}=0$ then $\mathscr{M}_{m_{*}, n}$ lies in $\mathscr{M}_{g, n}^{\text {comb }}$, for some $g$, in an evident way. One can check that $\mathscr{M}_{m_{*}, n}$ is a cycle with closed support on $\overline{\mathscr{M}}_{g, n} \times \mathbf{R}_{+}^{n}$. Using the isomorphism

$$
H_{*}^{\text {closed }}\left(\overline{\mathscr{M}}_{g, n} \times \mathbf{R}_{+}^{n}\right) \cong H_{*-n}\left(\overline{\mathscr{M}}_{g, n}\right)
$$

and Poincaré duality with rational coefficients we obtain cohomology classes on $\overline{\mathscr{M}}_{g, n}$. They are even-dimensional; for any $k$ we obtain $p(k)$ classes in degree $2 k$, where $p(k)$ is the number of partitions of $k$.

Conjecture 3.4. All these classes can be expressed through Mumford-Miller classes (see $[\mathrm{M}, \mathrm{H}])$.

Recently R. Penner [P3] proved that the cycle $\mathscr{M}_{0,4 g-7+2 n, 0, \ldots, n}$ is Poincare dual of the first Mumford-Miller class $k_{1}$.

\section{Airy Functions}

4.1. The Classical and the Matrix Airy Functions. The classical Airy function

$$
A(y)=\int_{-\infty}^{\infty} \exp \left(\sqrt{-1}\left(x^{3} / 3-x y\right)\right) d x
$$

is the unique (up to scalar factor) bounded solution of the differential equation

$$
A^{\prime \prime}(y)+y \cdot A(y)=0 .
$$

We define the matrix Airy function to be

$$
A(Y)=\int \exp \left(\sqrt{-1} \operatorname{tr}\left(X^{3} / 3-X Y\right)\right) d X,
$$

where $X, Y$ are hermitian $N \times N$ matrices for some $N$. The function $A(Y)$ is well-defined as a distribution. It obeys an elliptic equation

$$
\Delta A(Y)+\operatorname{tr} Y \cdot A(Y)=0,
$$

where $\Delta$ is the Laplacian. Hence $A$ is a smooth function.

The asymptotic expansion for $A(Y)$ as $Y \rightarrow+\infty$ is the sum of terms corresponding to the critical points of the function $\operatorname{tr}\left(X^{3} / 3-X Y\right)$ (the method of stationary phase). Suppose that all eigenvalues of $Y$ are distinct. Then the critical points are all $2^{N}$ square roots of $Y$ and the corresponding summands are

$$
\begin{aligned}
& \int_{\text {near } Y^{1 / 2}} \exp \left(\sqrt{-1} \operatorname{tr}\left(X^{3} / 3-X Y\right) d X\right. \\
& \quad=\int_{\text {near } 0} \exp \left(\sqrt{-1} \operatorname{tr}\left(\left(X+Y^{1 / 2}\right)^{3} / 3-\left(X+Y^{1 / 2}\right) Y\right)\right) d X \\
& \quad=\exp \left(-\frac{2 \sqrt{-1}}{3} \operatorname{tr} Y^{3 / 2}\right) \times \int_{\text {near } 0} \exp \left(\sqrt{-1} \operatorname{tr}\left(X^{3} / 3-X^{2} Y^{1 / 2}\right)\right) d X .
\end{aligned}
$$


The asymptotic expansion for the last integral is given essentially by Theorem 1.1 (the fact that $\Lambda=2 \sqrt{-1} Y^{1 / 2}$ is not hermitian is not a serious obstruction). We obtain the formula

$$
\begin{aligned}
A(Y) \sim \sum_{Y^{1 / 2}} \exp \left(-\frac{2 \sqrt{-1}}{3} \operatorname{tr} Y^{3 / 2}\right) & \times \operatorname{det}\left(\frac{\sqrt{-1}}{\pi}\left(Y^{1 / 2} \otimes 1+1 \otimes Y^{1 / 2}\right)\right)^{-1 / 2} \\
& \times \exp \left(F\left(\tilde{t}_{*}\left(Y^{1 / 2}\right)\right)\right)
\end{aligned}
$$

where $\tilde{t}_{i}\left(Y^{1 / 2}\right)=2^{-(2 i+1) / 3}(2 i-1) ! ! \operatorname{tr} Y^{-i-1 / 2}$ and the square root of the matrix $\frac{\sqrt{-1}}{\pi}\left(Y^{1 / 2} \otimes 1+1 \otimes Y^{1 / 2}\right)$ is chosen to have eigenvalues with positive real parts.

We recall the formula of Harish-Chandra (see $[\mathrm{HC}]$ ):

Lemma 4.1. If $\Phi$ is a conjugacy invariant function on the space of hermitian $N \times N$-matrices, then for any diagonal hermitian matrix $Y$,

$$
\begin{aligned}
& \int \Phi(X) e^{-\sqrt{-1} \operatorname{tr} X Y} d X \\
& \quad=(-2 \pi \sqrt{-1})^{N(N-1) / 2}(V(Y))^{-1} \int \Phi(D) e^{-\sqrt{-1} \operatorname{tr} D Y} V(D) d D,
\end{aligned}
$$

where the last integral is taken over the space of diagonal hermitian matrices $D$,

$$
V\left(\operatorname{diag}\left(X_{1}, \ldots, X_{N}\right)\right):=\prod_{i<j}\left(X_{j}-X_{i}\right)=\operatorname{det}\left(X_{i}^{j-1}\right)
$$

is the Vandermonde determinant.

This formula was recovered several times (see [IZ] and [Mh]). We apply the formula of Harish-Chandra to the case $\Phi(X)=\exp \left(\sqrt{-1} \operatorname{tr} X^{3} / 3\right)$. Some difficulties arise because integrals are not absolutely convergent. Nevertheless we obtain that the exact formula, $Y=\operatorname{diag}\left(Y_{1}, \ldots, Y_{N}\right)$ :

$$
\begin{aligned}
A(Y) & =(-2 \pi \sqrt{-1})^{N(N-1) / 2}(V(Y))^{-1} \int \cdots \int \prod_{i} e^{\sqrt{-1}\left(X_{i}^{3} / 3-X_{i} Y_{i}^{1 / 2}\right)} \operatorname{det}\left(X_{i}^{j-1}\right) d X_{i} \\
& =(2 \pi)^{N(N-1) / 2} \frac{\operatorname{det}\left(A^{(j-1)}\left(Y_{i}\right)\right)}{\operatorname{det}\left(Y_{i}^{j-1}\right)} .
\end{aligned}
$$

We use here the obvious fact that

$$
\int e^{\sqrt{-1}\left(x^{3} / 3-x y\right)} x^{j-1} d x=\left(\sqrt{-1} \frac{\partial}{\partial y}\right)^{j-1} A(y) .
$$

4.2. The $\tau$-Function Related with the Airy Function. Recall that $\tau$-functions for the KP-hierarchy (see [SW]) are functions (or formal series) in an infinite number of variables $T_{1}, T_{2}, \ldots$ satisfying a certain infinite system of nonlinear differential equations of infinite order (Hirota bilinear equations). A $\tau$-function for the KdV-hierarchy is a $\tau$-function which does not depend on variables $T_{1}, T_{2}, \ldots$. The logarithm of such a $\tau$-function satifies the $\mathrm{KdV}$-equation (see Sect. 1.2) with respect to the variables $T_{1}$ and $T_{3}$.

The definition of the $\tau$-function is the following: 
Let $V \subset \mathbf{C}((z))$ be an infinite dimensional linear subspace with a base $f_{1}, f_{2}, \ldots, f_{i}=z^{-i}(1+o(1))$, for almost all $i$ ( $V$ belongs to Sato's Grassmanian). For an infinite sequence of formal variables $T_{i}$ denote by $M\left(T_{*}\right)$ the operator of multiplication by the function $\exp \left(\sum_{i=1}^{\infty} T_{i} z^{-i} / i\right)$. The $\tau$-func-
tion associated to the subspace $V$ is

$$
\tau_{f_{*}}\left(T_{*}\right)=\left(M\left(T_{*}\right)\left(\cdots \wedge f_{3} \wedge f_{2} \wedge f_{1}\right) \wedge z^{0} \wedge z^{1} \wedge z^{2} \wedge \ldots\right): \bigwedge_{i=-\infty}^{+\infty} z^{i} .
$$

This function depends (up to a multiple) only on the subspace $V$. Formally $\tau_{f_{*}}\left(T_{*}\right)=0$ iff $M\left(T_{*}\right)(V) \cap \mathbf{C}[[z]] \neq 0$.

We will use the following "explicit" formula for $\tau$-functions:

Lemma 4.2. Let $f_{1}, f_{2}, \ldots \in \mathbf{C}((z))$ be formal Laurent series, $f_{i}=z^{-i}(1+o(1))$. Then, for any $N \geqq 0$, the following symmetric series in $n$ variables $z_{1}, \ldots, z_{N}$

(1) $\operatorname{det}\left(f_{i}\left(z_{j}\right)\right) / \operatorname{det}\left(z_{j}^{-i}\right)$,

(2) $\tau_{f_{*}}\left(T_{1}\left(z_{*}\right), T_{2}\left(z_{*}\right), \ldots\right)$, where $T_{k}\left(z_{*}\right)=\frac{1}{k} \sum_{i=1}^{N} z_{i}^{k}$

coincide. All $\tau$-functions such that $\tau(0,0, \ldots)=1$ can be obtained in this way.

Sketch of the proof. First of all, note that

$$
\left(\exp \sum_{i=1}^{\infty} T_{i}\left(z_{*}\right) z^{-i} / i\right)=\left(\prod_{i=1}^{N}\left(1-z_{i} / z\right)\right)^{-1}
$$

Hence (formally) $\tau_{f_{*}}\left(T_{1}\left(z_{*}\right), T_{2}\left(z_{*}\right), \ldots\right)=0$ iff

$$
V \cap \prod_{i=1}^{N}\left(1-z_{i} / z\right) \mathbf{C}[[z]] \neq 0 .
$$

Any nonzero element in this intersection is a nontrivial linear combination of functions $f_{1}, f_{2}, \ldots, f_{N}$, which vanishes at points $z_{1}, \ldots, z_{N}$. We conclude that $\tau_{f_{*}}\left(T_{1}\left(z_{*}\right), T_{2}\left(z_{*}\right), \ldots\right)$ is divisible by $\operatorname{det}\left(f_{i}\left(z_{j}\right)\right) / \operatorname{det}\left(z_{j}^{-i}\right)$. For reasons of degree this ratio is a constant.

Any subspace transversal to $\mathbf{C}[[z]]$ admits a base $f_{i}$ with $f_{i}=$ $z^{-i}(1+o(1))$.

Let us now return to Airy functions. It is easy to see that there exists an asymptotic expansion for derivatives of the (ordinary) Airy function:

$$
A^{(j-1)}(y) \sim \sum_{ \pm \sqrt{y}} \text { const } \cdot y^{-3 / 4} e^{-\frac{2 \sqrt{-1}}{3} y^{3 / 2}} \cdot f_{j}\left(y^{-1 / 2}\right)
$$

for some series $f_{j}(z)=z^{-j}+\cdots \in \mathbf{Q}((z))$. Substitution of the last formula to (2) gives

$$
\sum_{Y^{1 / 2}} \text { const } \times \exp \left(-\frac{2 \sqrt{-1}}{3} \operatorname{tr} Y^{3 / 2}\right) \times \prod_{i=1}^{N} Y_{i}^{-3 / 4} \times \operatorname{det}\left(f_{j}\left(Y_{i}^{-1 / 2}\right)\right) / \operatorname{det}\left(Y_{i}^{j-1}\right) \text {. }
$$


Proof of Theorem 1.2. We can compare asymptotic expansions (1), (2), and (3) and obtain that $F\left(t_{*}\right)$ is a $\tau$-function for KdV-hierarchy in variables

$$
T_{2 i+1}=2^{(2 i+1) / 3} t_{i} /(2 i+1) ! !
$$

The class of $\tau$-functions is stable under the change of variables $T_{i} \mapsto c^{i} T_{i}$ for any constant $c$. We set $c:=2^{-2 / 3}$.

Remark. With any $\tau$-function $T$ we associate (under appropriate convergence conditions) a function on matrices of arbitrary size:

$$
T(X):=T\left(\operatorname{tr} X, \operatorname{tr} X^{2} / 2, \ldots\right) .
$$

The transform $X \rightarrow T_{k}:=\operatorname{tr} X^{k} / k$ is called the passing to Miwa's coordinates.

One can try to combine Lemma 4.1 and Lemma 4.2 and obtain examples of (matrix) $\tau$-functions such that their Fourier transform is again (up to a simple factor and some change of variables) a matrix $\tau$-function. The matrix $\tau$-function associated with the subspace

$$
\left\langle\exp \left(\sqrt{-1} z^{3} / 3\right) z^{-1}, \exp \left(\sqrt{-1} z^{3} / 3\right) z^{-2}, \ldots\right\rangle
$$

is $\exp \left(\sqrt{-1} \operatorname{tr} X^{3} / 3\right)$. Other examples are the higher Airy functions (see the next section). They were proposed independently by M. Adler and P. van Moerbeke ([AvM]) and by a Moscow group of physicists ([KMMMZ]). The last group also considered a generalization to the case of Fourier transforms of functions of the form $\exp (\operatorname{tr}(F(x)))$, where $F$ is arbitrary function in one variable.

Morally, the combination of Lemma 4.1 and Lemma 4.2 means that Fourier transform preserves the class of matrix $\tau$-functions. We will discuss transformations of matrix $\tau$-functions elsewhere ([KM]).

We propose the following generalization of the Conjecture 3.1:

Conjecture 4.1. Let $T$ be any formal $\tau$-function for the KdV-hierarchy considered as a matrix function. Then $\int T(X) d \mu_{\Lambda}(X)$ is a matrix $\tau$-function for the $\mathrm{KdV}$-hierarchy in $\Lambda$.

4.3. Higher Airy Functions. Define the higher Airy function for $d \geqq 2$ to be

$$
A_{d}(y)=\int_{-\infty}^{\infty} \exp \left(\sqrt{-1}\left(x^{d+1} /(d+1)-x y\right)\right) d x .
$$

We can also define a higher matrix Airy function. The same arguments as in Sects. 4.1, 4.2 give an asymptotic expansion for the higher matrix Airy function in terms of some $\tau$-function for KP-hierarchy. This $\tau$-function does not depend on the variables $T_{d}, T_{2 d}, T_{3 d} \ldots$ (this is connected with the $d^{\text {th }}$ analog of the KdV-equation) and homogeneity degree of each monomial is divisible by $d+1$. One can compare this function with the solution of some modification of the standard matrix model, called chain of matrices or $(d-1)$ matrix model or $W_{d}$-gravity. It turns out that these $\tau$-functions coincide (we use formulas from [KS]). Recently E. Witten proposed the following algebro-geometric description of corresponding higher gravity: 
Denote by $\overline{\mathscr{M}}_{g, n, r_{*}}^{\prime}$, for $0 \leqq r_{1}, \ldots, r_{n} \leqq d-2$, the moduli space of stable complex curves $C$ of genus $g$ with $n$ distinct marked points $x_{1}, \ldots, x_{n}$, with a line bundle $\mathscr{R}$ on $C$ and with an isomorphism

$$
\mathscr{R}^{\otimes d} \cong K^{d-1} \otimes \bigotimes_{i=1}^{n} \mathcal{O}\left(x_{i}\right)^{\otimes r_{i}},
$$

where $K$ is the canonical bundle (= cotangent bundle for smooth curves) and $\mathcal{O}\left(x_{i}\right)$ is the line bundle whose sections are functions with a possible pole at $x_{i}$.

On $\overline{\mathscr{M}}_{g, s, r_{*}}^{\prime}$ one gets a vector bundle $\mathscr{V}$ with fibers equal to $H^{0}(C, \mathscr{R})$. Denote by $c_{T}(\mathscr{V})$ its top Chern class (= Euler class). If $d=2$ then $\operatorname{dim} \mathscr{V}=0$, $c_{T}(\mathscr{V})=1$. For an infinite sequence of indeterminates $\tau_{k}\left(U_{r}\right), r=0,1, \ldots$, $d-2, n=0,1, \ldots$, define brackets

$$
\left\langle\tau_{k_{1}}\left(U_{r_{1}}\right) \ldots \tau_{k_{n}}\left(U_{r_{n}}\right)\right\rangle=\frac{1}{d^{g}} \int_{\bar{M}_{g, s, r_{*}}^{\prime}} c_{T}(\mathscr{V}) \cdot \prod_{i=1}^{n} c_{1}\left(\mathscr{L}_{i}\right)^{k_{i}} .
$$

The generating function for these numbers is

$$
F_{d}\left(t_{1}, t_{2}, \ldots\right)=\sum_{k_{*}, r_{*}}\left\langle\tau_{k_{1}}\left(U_{r_{1}}\right) \ldots \tau_{k_{n}}\left(U_{r_{n}}\right)\right\rangle \times \prod t_{d \times k_{i}+r_{i}+1} / n ! .
$$

Conjecture 4.2 (E. Witten, [W3]). $\exp \left(F_{d}\right)$ coincides with the $\tau$-function for the $(d-1)$ matrix model.

One can hope that it is possible to connect Feynman diagrams arising from the higher matrix Airy functions to the topology of $\overline{\mathscr{M}}_{g, s, r_{*}}^{\prime}$.

\section{Appendix A. Orbispaces}

W. Thurston proposed several years ago the term orbifold for a space which looks locally like a factor space of a manifold modulo an action of a finite group. Before W. Thurston, I. Satake used the term V-manifolds.

If an infinite discrete group acts properly discontinuous on a manifold then the factor space is an orbifold. For example the moduli space of curves $\mathscr{M}_{g, n}$ is an orbifold because it is the factor space of Teichmüller space modulo the action of the mapping class group. The Deligne-Mumford compactification $\overline{\mathscr{M}}_{g, n}$ is the example of an orbifold which has no global covering manifold.

We will use the name orbispace for the extension of the notion of orbifold to the nonsmooth case. Roughly speaking the structure of orbispace on a topological space $X$ is a semicontinuous way of attaching to each point $x \in X$ a finite group $\Gamma_{x}$ defined up to an inner automorphism. Locally this family $\Gamma_{x}$ must look like the following basic example: If a finite group $G$ acts on a topological space $Y$ then with each point $x \in X:=Y / G$ one can associate an isomorphism class of group $\mathrm{Stab}_{y}$ for any point $y$ from the orbit $x$. Moreover, for any two points $y, y^{\prime} \in x$, there is a natural isomorphism $\mathrm{Stab}_{y} \cong \mathrm{Stab}_{y}^{\prime}$ defined only up to a multiplication by an inner isomorphism.

We will not give a precise definition of orbispaces, (it uses nonabelian cohomology), say only that an orbispace is a particular example of a stack, see $[\mathrm{M}]$. 
The moduli space of ribbon graphs from Theorem 2.2 carries the natural structure of an orbispace, because the automorphism group of any ribbon graph with metric is finite.

One can extend many usual notions to the world of orbispaces, for example, vector bundles, homotopy type, differential forms. The cohomology groups of an orbispace with rational coefficients coincide with the cohomology groups of its underlying topological space. For an orientable compact orbifold $X$ the fundamental class as an element of $H_{*}(X, \mathbf{Q})$ is defined.

Consider for example the orbifold \{point $/ G$, where $G$ is a finite group acting trivially on the one-point space. Vector bundles on this orbispace are representations of $G$, the homotopy type is the Eilenberg-Mac Lane space $K(G, 1)$ and the fundamental class is $1 / \# G \in \mathbf{Q} \cong H_{0}($ point $\left.\}, \mathbf{Q}\right)$.

\section{Appendix B. More about Topology on $\mathscr{M}_{g, n}^{\text {comb }}$}

For a ribbon graph $\Gamma$, denote by $\mathscr{M}_{\Gamma}$ the set of functions $l: X_{1} \rightarrow \mathbf{R}_{\geq 0}$ such that there is no nontrivial cycle $e_{1}, \ldots, e_{n}$ with $l\left(e_{i}\right)=0$ for all $i$; denote by $\overline{\mathscr{M}}_{\Gamma}$ the set of functions $l \geqq 0$ such that any boundary component contains an edge $e$ with $l(e)>0$.

$\mathscr{M}_{\Gamma} \subset \overline{\mathscr{M}}_{\Gamma}$ are open subsets of $\mathbf{R}_{\geqq 0}^{X_{1}}$, we endow them with the induced topology. If a ribbon graph $\Gamma^{\prime}$ is obtained from $\Gamma$ by the contraction of some edge $e \in X_{1}$ which is not a loop, then there are natural embeddings:

$$
\mathscr{M}_{\Gamma^{\prime}} \hookrightarrow \mathscr{M}_{\Gamma}, \quad \overline{\mathscr{M}}_{\Gamma^{\prime}} \hookrightarrow \overline{\mathscr{M}}_{\Gamma} \quad(\text { pose } l(e)=0) .
$$

Let us consider the following category $\Gamma_{g, n}$ :

- objects of $\Gamma_{g, n}$ are connected numbered ribbon graphs without vertices of valencies 1, 2 such that the corresponding surface has genus $g$ and $n$ holes,

- morphisms of $\Gamma_{g, n}$ are maps of abstract graphs generated by isomorphisms and contractions of edges preserving numbering.

$\mathscr{M}_{\Gamma}$ and $\overline{\mathscr{M}}_{\Gamma}$ can be considered as contravariant functors from $\Gamma_{g, n}$ to the category of topological spaces. Define $\mathscr{M}_{g, n}^{\text {comb }}$ and $\overline{\mathscr{M}}_{g, n}^{\text {comb }}$ to be the limits of functors $\mathscr{M}$ and $\overline{\mathscr{M}}$. Any point of $\mathscr{M}_{g, n}^{\text {comb }}$ has a unique (up to an isomorphism) representative $(\Gamma, l)$ such that $l(e)>0$ for all edges $e$. Hence we obtain the same set as in the definition in Sect. 2.1.

Spaces $\mathscr{M}_{g, n}^{\text {comb }}$ and $\overline{\mathscr{M}}_{g, n}^{\text {comb }}$ are locally compact, and the perimeter map

$$
\pi: \overline{\mathscr{M}}_{g, n}^{\text {comb }} \rightarrow \mathbf{R}_{+}^{n}
$$

is proper. The map $\mathscr{M}_{g, n}^{\text {comb }} \rightarrow\left(B U(1)^{\text {comb }}\right)^{n}$ (see Theorem 2.3) extends continuously to $\overline{\mathscr{M}}_{g, n}^{\text {comb }}$.

We will need a detailed description of points of $\overline{\mathscr{M}}_{g, n}^{\text {comb }}$.

Let us call a stable graph a ribbon graph $\Gamma$ together with some additional data:

(1) a subset $S$ of $X_{0}$ containing all vertices of valency 1 and 2;

(2) an equivalence relation $\sim$ on $S$;

(3) a function $g: S / \sim \rightarrow Z_{\geqq 0}$. 
This data must satisfy the following conditions:

(1) for each equivalence class $S^{\prime} \subset S$ the inequality $2-2 g\left(S^{\prime}\right)-\# S^{\prime}<0$ holds,

(2) the abstract graph obtained from $\Gamma$ after the identification of vertices by $\sim$, is connected.

One can show that $\overline{\mathscr{M}}_{g, n}^{\text {comb }}$ is the set of equivalence classes of numbered stable graphs with metric such that

$$
2-2 g-n=\# X_{0}-\# X_{1}-2 \# S+\sum_{S^{\prime} \in S / \sim}\left(2-2 g\left(S^{\prime}\right)\right) .
$$

An analytic analogue of $\overline{\mathscr{M}}_{g, n}^{\text {comb }}$ is a certain factor space $\overline{\mathscr{M}}_{g, n}^{\prime}$ of $\overline{\mathscr{M}}_{g, n}$. Consider the following equivalence relation on $\overline{\mathscr{M}}_{g, n}$ : two stable curves with marked points $\left(C, x_{1}, \ldots, x_{n}\right)$ and $\left(C^{\prime}, x_{1}^{\prime}, \ldots, x_{n}^{\prime}\right)$ are equivalent if there exists a homeomorphism $\phi: C \rightarrow C^{\prime}$ such that for any $i \phi\left(x_{i}\right)=x_{i}^{\prime}$ and $\phi$ is complex analytic on all components containing marked points. Define $\overline{\mathscr{M}}_{g, n}^{\prime}$ to be the factor space of $\overline{\mathscr{M}}_{g, n}$ modulo the closure of this relation.

Using Strebel results (Theorem 2.1), for the noncompact case one can construct a natural bijective map

$$
\overline{\mathscr{M}}_{g, n}^{\text {comb }} \rightarrow \overline{\mathscr{M}}_{g, n}^{\prime} \times \mathbf{R}_{+}^{n} .
$$

The main technical point, which will be omitted here, is that this map is continuous. From this fact and the facts that $\overline{\mathscr{M}}_{g, n}^{\text {comb }}$ is proper over $\mathbf{R}_{+}^{n}$ and $\overline{\mathscr{M}}_{g, n}^{\prime}$ is compact, one deduces immediately that $\overline{\mathscr{M}}_{g, n}^{\text {comb }}$ is homeomorphic to $\bar{M}_{g, n}^{\prime} \times \mathbf{R}_{+}^{n}$ and (as the consequence) $\mathscr{M}_{g, n}^{\text {comb }}$ is homeomorphic to $\mathscr{M}_{g, n} \times \mathbf{R}_{+}^{n}$.

\section{Appendix C. Computation of the Constant $\varrho$}

Let $\Gamma \in \mathscr{M}_{m_{*}, n}$ be a connected ribbon graph which has no vertices with even valency. Consider the following complex of free abelian groups:

$$
\begin{aligned}
& C^{i}=0, \quad i \leqq 0 \text { or } i \geqq 5, \\
& C^{1}=C^{4}=\mathbf{Z}^{X_{2}}, \\
& C^{2}=C^{3}=\mathbf{Z}^{X_{1}},
\end{aligned}
$$

with differentials defined by the formulas

$$
\begin{aligned}
& d_{1}\left([x]_{2}\right)=\left[x_{1}\right]+\left[s_{2} x\right]_{1}+\cdots+\left[s_{2}^{\#[x]_{2}} x\right]_{1}, \\
& d_{2}\left([x]_{1}\right)=\sum_{j=1}^{\#[x]_{0}-1}(-1)^{j}\left[s_{0}^{j} x\right]_{1}+\sum_{j=1}^{\#\left[s_{1} x\right]_{0}-1}(-1)^{j}\left[s_{0}^{j} s_{1} x\right]_{1}, \\
& d_{3}\left([x]_{1}\right)=[x]_{2}+\left[s_{1} x\right]_{2} .
\end{aligned}
$$

We can identify $C^{*} \otimes \mathbf{R}$ with the complex

$$
T^{*} \mathbf{R}_{+}^{n} \stackrel{T^{*} \pi}{\longrightarrow} T_{\Gamma}^{*} \mathscr{M}_{m_{*}, n} \stackrel{\beta}{\longrightarrow} T_{\Gamma} \mathscr{M}_{m_{*}, n} \stackrel{T \pi}{\longrightarrow} T \mathbf{R}_{+}^{n} .
$$

Here $\beta$ is a 2 -vector field with constant coefficients on $\mathscr{M}_{m_{*}, n}$ given by the formula for $d_{2}$. 
The following simple lemmas describe the geometry of $\beta$. The (straightforward) proofs are omitted.

Lemma C.1. The kernel of $\beta$ coincides with the image of $T^{*} \pi$. The map $T^{*} \pi$ is injective.

Hence the symplectic fibers of $\beta$ coincide with fibers of $\pi$ and complex $C^{*} \otimes \mathbf{R}$ is acyclic.

Lemma C.2. The symplectic form on fibers of $\pi$ coincides with the restriction of $\Omega / 4$.

We have the canonical (up to sign) volume form on each term of the acyclic complex $C^{*} \otimes \mathbf{R}$ : the Lebesgue measure.

One can easily define the canonical isomorphism

$$
\underset{i}{\otimes}\left(\wedge^{\operatorname{dim} A^{i}} A^{i}\right)^{\otimes(-1)^{i}} \stackrel{\sim}{\longrightarrow} \underset{i}{\otimes}\left(\wedge^{\operatorname{dim} H^{i}\left(A^{*}\right)} H^{i}\left(A^{*}\right)\right)^{\otimes(-1)^{i}}
$$

for any finite dimensional complex $A^{*}$. Applying this isomorphism to $C^{*} \otimes \mathbf{R}$, we obtain a measure on $\mathbf{R}$. This measure is the product of the Lebesgue measure by a positive real number called the torsion of the complex $C^{*} \otimes \mathbf{R}$.

From Lemma C.2 it follows that the torsion is equal to $4^{d} \varrho$. The canonical measure on $C^{*} \otimes \mathbf{R}$ has the property that $\operatorname{vol}\left(C^{*} \otimes \mathbf{R} / C^{*}\right)=1$. One can deduce easily that

$$
\varrho=4^{d} \times\left(\prod_{i}\left(\# H^{i}\left(C^{*}\right)\right)^{(-1)^{i}}\right)^{1 / 2} .
$$

Theorem C.1. Let $C$ be the closed surface associated with $\Gamma$. There is an isomorphism $H^{i}\left(C^{*}\right) \cong H^{i-2}(C, \mathbf{Z} / 2 \mathbf{Z})$.

Corollary. $\varrho=4^{d} \times 2^{1-g}$.

The proof of Theorem C.1 presented here is not nice, but we don't know any other proof.

Lemma C.3. For any $k$ and any $\xi \in C^{k}$, if $d_{k} \xi=0$ then there exists $\eta \in C^{k-1}$ such that $d_{k-1} \eta=2 \xi$.

Proof. If $k=1$ and $d_{1} \xi=0$ then $\xi=0$, because all valencies are odd.

In the case $k=2$ we can use Lemma C. 2 to construct $\eta$ explicitly.

Let us choose for each boundary component $b \in X_{2}$ some element $f(b) \in b$. For any $x \in X$ denote by $v(x)$ the minimal integer $i, 1 \leqq i \leqq \#[x]_{2}$ such that $s_{2}^{i} x=f\left([x]_{2}\right)$.

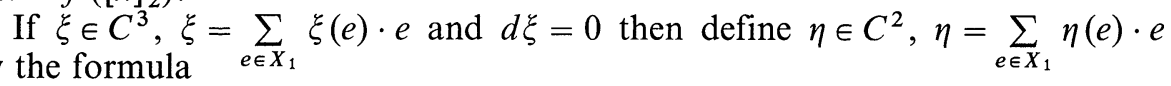

$$
\eta\left([x]_{1}\right)=\xi\left([x]_{1}\right)+\sum_{i=2}^{v(x)} \xi\left(\left[s_{2}^{i} x\right]_{1}\right)+\sum_{i=2}^{v\left(s_{1} x\right)} \xi\left(\left[s_{2}^{i} s_{1} x\right]_{1}\right) .
$$

If $\xi \in C^{4}, \xi=b$ for some boundary component, then

$$
\eta=\#\left[\sum_{i=0}^{(b)]_{1}-1}(-1)^{i}\left[s_{0}^{-i} f(b)\right]_{1} .\right.
$$


An easy consequence of the statement of Lemma C.3 is the fact that $C^{*}$ can be decomposed into a direct sum of complexes of types $\mathbf{Z} \stackrel{1}{\longrightarrow} \mathbf{Z}$ and $\mathbf{Z} \stackrel{2}{\longrightarrow} \mathbf{Z}$. The cohomology groups $H^{i}\left(C^{*}\right)$ are vector spaces over the field $\mathbf{F}_{2}$. Let $\tau_{i}=\operatorname{dim}_{\mathbf{F}_{2}} H^{i}\left(C^{*}\right)$, then $\operatorname{dim}_{\mathbf{F}_{2}} H^{i}\left(C^{*} \otimes \mathbf{Z} / 2 \mathbf{Z}\right)=\tau_{i}+\tau_{i-1}$. Hence we need to compute dimensions of $H^{i}\left(C^{*} \otimes \mathbf{Z} / 2 \mathbf{Z}\right)$.

The complex $C^{*} \otimes \mathbf{Z} / 2 \mathbf{Z}$ is obtained by glueing the two complexes

$$
\begin{aligned}
& A^{*}: 0 \rightarrow \mathbf{F}_{2}^{X_{2}} \stackrel{\partial_{2}}{\longrightarrow} \mathbf{F}_{2}^{X_{1}} \stackrel{\partial_{1}}{\longrightarrow} \mathbf{F}_{2}^{X_{0}} \rightarrow 0 \text { and } \\
& B^{*}: 0 \rightarrow \mathbf{F}_{2}^{X_{0}} \stackrel{\partial_{2}^{*}}{\longrightarrow} \mathbf{F}_{2}^{X_{1}} \stackrel{\partial_{*}^{*}}{\longrightarrow} \mathbf{F}_{2}^{X_{2}} \rightarrow 0 .
\end{aligned}
$$

$A^{*}$ is the chain complex and $B^{*}$ is the cochain complex with $\mathrm{F}_{2}$ coefficients for the cell decomposition of the surface $C$ determined by the graph $\Gamma$.

The differentials of $C^{*} \otimes \mathbf{Z} / 2 \mathbf{Z}$ are $d_{1}=\partial_{2}, d_{2}=\partial_{0}^{*} \partial_{1}, d_{3}=\partial_{1}^{*}$.

$$
\begin{aligned}
& H^{1}\left(C^{*} \otimes \mathbf{Z} / 2 \mathbf{Z}\right) \cong H^{0}\left(A^{*}\right) \cong H_{2}\left(C, \mathbf{F}_{2}\right) \cong \mathbf{F}_{2}, \\
& H^{4}\left(C^{*} \otimes \mathbf{Z} / 2 \mathbf{Z}\right) \cong H_{2}\left(B^{*}\right) \cong H^{2}\left(C, \mathbf{F}_{2}\right) \cong \mathbf{F}_{2} .
\end{aligned}
$$

Let us compute the dimension of $H^{3}\left(C^{*} \otimes \mathbf{Z} / 2 \mathbf{Z}\right)$ :

Lemma C.4. 1) Codim $\operatorname{Im} \partial_{1}=1$, 2) $\operatorname{Ker} \partial_{0}^{*} \subset \operatorname{Im} \partial_{1}$.

Proof. Part 1) is evident.

It is easy to see that Ker $\partial_{0}^{*}$ consists in two elements -0 and

$$
\sum_{v \in X_{0}} v=\partial_{1} \sum_{e \in X_{1}} e .
$$

From Lemma C. 4 it follows that the space $\operatorname{Im} \partial_{0}^{*} \partial_{1}$ is a subspace of codimension 1 in the space $\operatorname{Im} \partial_{0}^{*}$. Hence

$$
\operatorname{dim}_{\mathbf{F}_{2}} H^{3}\left(C^{*} \otimes \mathbf{Z} / 2 \mathbf{Z}\right)=\operatorname{dim}_{\mathbf{F}_{2}} H^{2}\left(C, \mathbf{F}_{2}\right)+1=2 g+1,
$$

and $\tau_{1}=0, \tau_{2}=1, \tau_{3}=2 g, \tau_{4}=1$. Thus Theorem C.1 is proven.

\section{Appendix D. A Short Computation of $\chi\left(\mathscr{M}_{g, n}\right)$}

For integers $g \geqq 0$ and $n>0$ denote by $\chi_{g, n}:=2-2 g-n$ the Euler characteristic of open curves of the corresponding type. Let us consider the following formal series:

$$
X(t):=\sum_{g, n: \chi_{g}, n} \frac{\chi\left(\mathscr{M}_{g, n}\right)}{n !} t^{\chi_{g, n}} .
$$

Here $\chi\left(\mathscr{M}_{g, n}\right)$ denotes the Euler characteristic of the moduli space of curves considered as an orbifold. For example, the coefficient of $t^{-1}$ in $X(t)$ is $\frac{\chi\left(\mathscr{M}_{0,3}\right)}{6}+\chi\left(\mathscr{M}_{1,1}\right)=\frac{1}{6}+\frac{-1}{12}=\frac{1}{12}$. We can rewrite $X(t)$ as a sum over the set of all equivalence classes of connected ribbon graphs with valencies greater than 2 using the stratification (Theorem 2.2). The codimension of the strata correspoding to any graph $\Gamma$ from $\mathscr{M}_{g, n}^{\text {comb }}$ is equal to $6 g-6+3 n-$ 
$\# X_{1}=\# X_{0}(\bmod 2)$. Hence the contribution of the graph $\Gamma$ is equal to $\frac{1}{\# \text { Aut } \Gamma}(-1)^{\# X_{0}} t^{\# X_{0}-\# X_{1}}$. We see that $X(t)$ is an asymptotic expansion of

$$
\log \left(\sqrt{\frac{t}{2 \pi}} \int \exp \left(-t \sum_{k=2}^{\infty} \frac{x^{k}}{k}\right) d x\right) .
$$

We take in this formula the integral over an arbitrary sufficiently small neighbourhood of 0 , the asymptotic expansion when $t \rightarrow+\infty$ does not depend on the choice of the neighbourhood. Further we will identify functions differ by a function rapidly decreasing at $+\infty$. The integral in the formula above is equal to

$$
\begin{gathered}
\int_{\text {near } 0} \exp (t(x+\log (1-x))) d x=\int(1-x)^{t} e^{t x} d x=e^{t} \int_{\text {near } 1} y^{t} e^{-t y} d y \\
=e^{t} \int_{0}^{\infty} y^{t} e^{-t y} d y=\frac{e^{t}}{t^{t+1}} \int_{0}^{\infty} z^{t} e^{-z} d z=\frac{e^{t}}{t^{t+1}} \Gamma(t+1) .
\end{gathered}
$$

Using the asymptotic expansion for the factorial we obtain the formula

$$
X(t)=\log \left(\frac{e^{t}}{t^{t} \sqrt{2 \pi t}} \Gamma(t+1)\right)=\sum_{k=1}^{\infty} \frac{\zeta(-k)}{-k} t^{-k} .
$$

This formula is well known, it is the specialization of Penner's model to the case of $1 \times 1$-matrices, see [P2].

Notice that $\chi\left(\mathscr{M}_{g, n+1}\right)=\chi\left(\mathscr{M}_{g, n}\right) \chi_{g, n}, \chi_{g, n+1}=\chi_{g, n}-1$, hence

$$
\frac{\left.\chi \Phi \mathscr{M}_{g, n+1}\right)}{(n+1) !} t^{\chi_{g}, n+1}=\frac{d}{d t}\left(\frac{1}{n+1} \frac{\chi\left(\mathscr{M}_{g, n}\right)}{n !} t^{\chi_{g}, n}\right) .
$$

Denote by $A_{0}(t)$ the function $t \log (t)-t$ and by $A_{1}(t)$ the series

$$
\sum_{g \geqq 1} \chi\left(\mathscr{M}_{g, 1}\right) t^{\chi_{g, 1}}, \quad A(t):=A_{0}(t)+A_{1}(t) .
$$

We can express $X(t)$ using the function $A(t)$ :

$$
\begin{aligned}
X(t) & =\sum_{g \geqq 1, n \geqq 1} \frac{\chi\left(\mathscr{M}_{g, n}\right)}{n !} t^{\chi_{g}, n}+\sum_{n \geqq 3} \frac{\chi\left(\mathscr{M}_{0, n}\right)}{n !} t^{\chi_{0, n}} \\
& =A_{1}(t)+A_{1}^{\prime}(t) / 2 !+A_{1}^{\prime \prime}(t) / 3 !+\cdots+A_{0}^{\prime \prime}(t) / 3 !+A_{0}^{\prime \prime \prime}(t) / 4 !+\cdots \\
& =\left(A(t)+A^{\prime}(t) / 2 !+A^{\prime \prime}(t) / 3 !+\ldots\right)-A_{0}(t)-A_{0}^{\prime}(t) / 2 \\
& =\int_{t}^{t+1} A(x) d x-t \log t+t-\frac{\log t}{2} .
\end{aligned}
$$

So, function $A(t)$ satisfies the equation

$$
\int_{t}^{t+1} A(x) d x=\log \left(\frac{\Gamma(t+1)}{\sqrt{2 \pi}}\right) .
$$

One can check that the function $A(x):=\frac{1}{2}-x+x \frac{d}{d x} \log \Gamma(x)$ is a solution of this equation. From the asymptotic formula for $\log \Gamma(x)$ we can deduce immediately the Harer-Zagier formula

$$
\chi\left(\mathscr{M}_{g, 1}\right)=\zeta(1-2 g) .
$$


Acknowledgements. I thank M.F. Atiyah, I. Kirchever, G. Segal, C.-F. Bödigheimer, D. Zagier, V. Kac and M. Mulase for useful discussions. Thanks to E. Witten for sending his preprints, and to R. Penner and C. Itzykson for their remarks. I am indebted to D. Zagier and E. Hironaka for their help in preparation of this paper. This work was done during my say in the Max-Planck-Institut für Mathematik. I am grateful to the Institute for the hospitality.

\section{References}

[AvM] Adler, M., van Moerbeke, P.: The $W_{p}$-gravity version of the Witten-Kontsevich model. Commun. Math. Phys. (to appear)

[BIZ] Bessis, D., Itzykson, C., Zuber, J.-B.: Qunatum field theory techniques in graphical enumeration. Adv. Appl. Math. 1, 109-157 (1980)

[BK] Brézin, E., Kazakov, V.: Lett B236, 144 (1990)

[DS] Douglas, M., Shenker, S.: Nucl. Phys. B335, 635 (1990)

[GM] Gross, D., Migdal, A.: Phys. Rev. Lett. 64, 127 (1990)

[H] Harer, J.: The cohomology of the moduli space of curves. Lecture Notes of mathematics, vol. 1337. Berlin, Heidelberg, New York: Springer, pp. 138-221

[HZ] Harer, J., Zagier, D.: The Euler characteristic of the moduli space of curves. Inv. Math. 85, 457-485 (1986)

[HC] Harish-Chandra: Differential operators on a semisimple Lie algebra. Am. J. Math. 79, 87-120 (1957)

[IZ1] Itzykson, C., Zuber, J.-B.: J. Math. Phys. 21, 411-421 (1980)

[IZ2] Itzykson, C., Zuber, J.-B.: Commun. Math. Phys. 134, 197-207 (1990)

[KS] Kac, V., Schwarz, A.: Lett. B257, 329 (1991)

[KMMMZ] Kharchev, S., Marshakov, A., Mironov, A., Morozov, A., Zabrodin, A.: Unification of all string models with $c<1$, FIAN/TD-9/91 and ITEP-M-8/91

[K1] Kontsevich, M.: Intersection theory on the moduli space of curves. Funct. Anal. and Appl. 25(2), 123-128 (1991)

[K2] Kontsevich, M.: Intersection theory on the moduli space of curves and the matrix Airy function. 30. Arbeitstagung Bonn. Preprint MPI/91-47

[KM] Kontsevich, M., Mulase, M.: In preparation

[Mh] Mehta, M.L.: Random matrices in nuclear physics and number theory. Contemporary Math. 50, 295-309 (1986)

[M] Mumford, D.: Towards an enumerative geometry of the moduli space of curves. In: Arithmetic and geometry, vol. II. Boston: Birkhäuser 1983

[P1] Penner, R.C.: The decorated Teichmüller space of punctured surfaces. Commun. Math. Phys. 113, 299-339 (1987)

[P2] Penner, R.C.: Perturbative series and the moduli space of Riemann surfaces. J. Diff. Geom. 27, 35-53 (1988)

[P3] Penner, R.C.: The Poincaré dual of the Weil-Petersson Kähler two-form. Preprint, October 1991

[SW] Segal, G. B., Wilson, G.: Loop groops and equations of KdV type. Publ. Math. I.H.E.S. 61, 5-65 (1985)

[S] Strebel, K.: Quadratic differentials. Berlin, Heidelberg, New York: Springer 1984

[W1] Witten, E.: Two dimensional gravity and intersection theory on moduli space. Surveys in Diff. Geom. 1, 243-310 (1991)

[W2] Witten, E.: On the Kontsevich model and other models of two dimensional gravity. IAS preprint HEP-91/24

[W3] Witten, E.: The $N$ matrix model and gauged WZW models. IAS preprint HEP$91 / 26$

[Z] Zwiebach, B.: How covariant closed string theory solves a minimal area problem. Commun. Math. Phys. 136, 83-118 
